\title{
Any Data Type
}

National Cancer Institute

\section{Source}

National Cancer Institute. Any Data Type. NCI Thesaurus. Code C95817.

A data type comprised of the basic properties of a data value. 